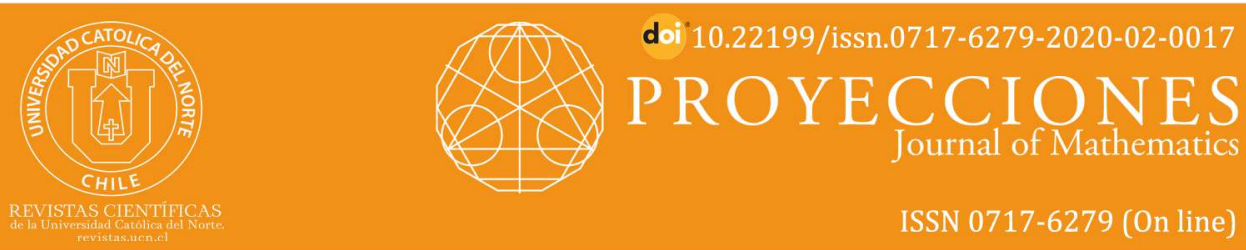

\title{
Restricted triangular difference mean graphs
}

\section{P. Jeyanthi ${ }^{1}$ (D) orcid.org/0000-0003-4349-164X \\ M. Selvi ${ }^{2}$ (i) orcid.org/0000-0002-6573-676X \\ D. Ramya ${ }^{3}$ (D) orcid.org/0000-0002-9261-4813}

${ }^{1}$ Govindammal Aditanar College for Women, Dept. of Mathematics, Tiruchendur, TN, India. 济 jeyajeyanthi@rediffmail.com

${ }^{2}$ Manonmaniam Sundaranar University, Research Scholar, No.12208, Tirunelveli, TN, India. selvm80@yahoo.in

${ }^{3}$ Government Arts College (Autonomous), Dept. of Mathematics, Salem, TN, India ॠaymar_padma@yahoo.co.in

Received: January 2019 | Accepted: November 2019

\section{Abstract:}

Let $G=(V, E)$ be a graph with $p$ vertices and $q$ edges. Consider an injection $f: V(G) \rightarrow\{1,2,3, \ldots, p q\}$. Define $f^{*}: E(G) \rightarrow$ $\left\{T_{1}, T_{2}, T_{3}, \ldots, T_{q}\right\}$, where $T_{q}$ is the $q^{\text {th }}$ triangular number such that $f^{*}(e)=\left\lceil\frac{|f(u)-f(v)|}{2}\right\rceil$ for all edges $e=u v$. If $f^{*}(E(G))$ is a sequence of consecutive triangular numbers $T_{1}, T_{2}, T_{3}, \ldots, T_{q}$, then the function $f$ is said to be restricted triangular difference mean. A graph that admits restricted triangular difference mean labeling is called restricted triangular difference mean graph. In this paper, we investigate restricted triangular difference mean behaviour of some standard graphs.

\section{Keywords: Restricted triangular difference mean labeling.}

\section{MSC (2010): 05C78}

\section{Cite this article as (IEEE citation style):}

P. Jeyanthi, M. Selvi, and D. Ramy, "Restricted triangular difference mean graphs", Proyecciones (Antofagasta, On line), vol. 39, no. 2, pp. 275-286, Apr. 2020, doi: 10.22199/issn.0717-62792020-02-0017.

Article copyright: (C) 2020 P. Jeyanthi, M. Selvi and D. Ramy. This is an open access article distributed under the terms of the Creative Commons Licence, which permits unrestricted use and distribution provided the original author and source are credited. 


\section{Introduction}

By a graph, we mean a finite, simple and undirected one. The vertex set and the edge set of a graph $G$ are denoted by $V(G)$ and $E(G)$ respectively. Terms and notations not defined here are used in the sense of Harary[1]. A graph labeling is an assignment of integers to the vertices or edges or both, subject to certain conditions. There are several types of labeling and a detailed survey is available in [2].

The concept of triangular difference mean labeling was introduced by Jeyanti et al.[3]. A triangular difference mean labeling of a graph $G=(p, q)$ is an injection $f: V(G) \rightarrow Z^{+}$, where $Z^{+}$is a set of positive integers such that for each edge $e=u v$, the edge labels are defined as $f^{*}(e)=$ $\left\lceil\frac{|f(u)-f(v)|}{2}\right\rceil$ such that the values of the edges are the first $q$ triangular numbers $T_{1}, T_{2}, \ldots, T_{q}$. A graph that admits restricted triangular difference mean labeling is called triangular difference mean graph.

In this paper we define a new labeling called restricted triangular difference mean labeling. Let $G=(V, E)$ be a graph with $p$ vertices and $q$ edges. Consider an injection $f: V(G) \rightarrow\{1,2,3, \ldots, p q\}$. Define $f^{*}$ : $E(G) \rightarrow\left\{T_{1}, T_{2}, T_{3}, \ldots, T_{q}\right\}$, where $T_{q}$ is the $q^{\text {th }}$ triangular number such that $f^{*}(e)=\left\lceil\frac{|f(u)-f(v)|}{2}\right\rceil$ for all edges $e=u v$. If $f^{*}(E(G))$ is a sequence of consecutive triangular numbers $T_{1}, T_{2}, T_{3}, \ldots, T_{q}$, then the function $f$ is said to be restricted triangular difference mean. A graph that admits restricted triangular difference mean labeling is called restricted triangular difference mean graph.

We use the following definitions in the subsequent sequel.

Definition 1.1. A vertex of degree one is called a pendant vertex and a pendant edge is an edge incident with a pendant vertex. The corona $G_{1} \odot G_{2}$ of the graphs $G_{1}$ and $G_{2}$ is obtained by taking one copy of $G_{1}$ (with $p$ vertices) and $p$ copies of $G_{2}$ and then join the $i^{\text {th }}$ vertex of $G_{1}$ to every vertex of the $i^{\text {th }}$ copy of $G_{2}$.

Definition 1.2. The bistar $B_{m, n}$ is a graph obtained from $K_{2}$ by joining $m$ pendant edges to one end of $K_{2}$ and $n$ pendant edges to the other end 
of $K_{2}$.

Definition 1.3. A triangular number is obtained by adding all positive integers less than or equal to a given positive integer $n$. If the $n^{\text {th }}$ triangular number is denoted by $T_{n}$, then $T_{n}=\frac{1}{2} n(n+1)$.

\section{Restricted Triangular Difference Mean graphs}

Theorem 2.1. Any path $P_{n}(n \geq 1)$ is a restricted triangular difference mean graph.

Proof. Let $v_{1}, v_{2}, \ldots, v_{n}$ be the vertices of the path $P_{n}$.

Then $E\left(P_{n}\right)=\left\{e_{i}=v_{i} v_{i+1}: 1 \leq i \leq n-1\right\}$.

Define $f: V\left(P_{n}\right) \rightarrow\{1,2,3, \ldots, p q\}$ is as follows:

$f\left(v_{1}\right)=1$,

$f\left(v_{2}\right)=2 T_{n-1}$,

$f\left(v_{i}\right)=f\left(v_{i-1}\right)+2(-1)^{i} T_{n-i+1}$ for $3 \leq i \leq n$.

For the vertex label $f$, the induced edge label $f^{*}$ is as follows:

$f^{*}\left(e_{i}\right)=T_{n-i}$ for $1 \leq i \leq n-1$. Hence $P_{n}$ is a restricted difference mean graph. The restricted triangular difference mean labeling of $P_{5}$ is given in Figure 1.

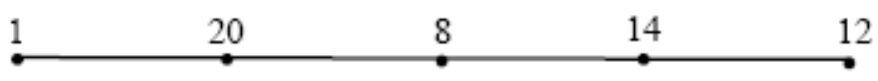

Figure 1

Theorem 2.2. The star graph $K_{1, n}(n \geq 1)$ admits restricted triangular difference mean labeling.

Proof. Let $v$ be the apex vertex and $v_{1}, v_{2}, \ldots, v_{n}$ be the pendant vertices of the star $K_{1, n}$. Then $E\left(K_{1, n}\right)=\left\{v v_{i}: 1 \leq i \leq n\right\}$. 
Define $f: V\left(K_{1, n}\right) \longrightarrow\{1,2,3, \ldots, p q\}$ as follows:

$f(v)=1, f\left(v_{i}\right)=2 T_{i}+1$ for $1 \leq i \leq n-1, f\left(v_{n}\right)=2 T_{n}$.

For the vertex label $f$, the induced edge label $f^{*}$ is as follows:

$f^{*}\left(v v_{i}\right)=T_{i}$ for $1 \leq i \leq n$. Hence, the induced edge labels are the first $\mathrm{n}$ triangular numbers. Therefore, $K_{1, n}$ is a restricted triangular difference mean graph. The restricted triangular difference mean labeling of $K_{1,5}$ is shown in Figure 2.

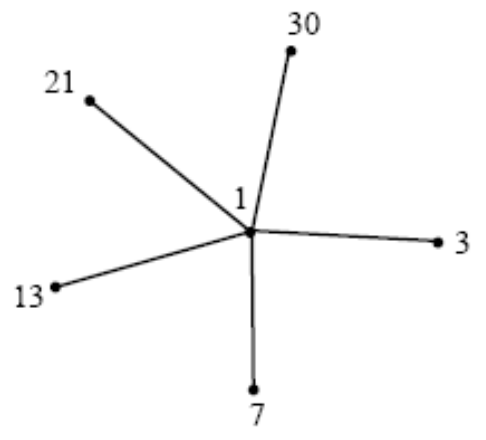

Figure 2

Theorem 2.3. The comb graph $P_{n} \odot K_{1}$ admits restricted triangular difference mean labeling.

Proof. Let $v_{1}, v_{2}, \ldots, v_{n}$ be the vertices of the path $P_{n}$ and $u_{1}, u_{2}, \ldots, u_{n}$ be the pendant vertices adjacent to $v_{1}, v_{2}, \ldots, v_{n}$ respectively. Then $E\left(P_{n} \odot\right.$ $\left.K_{1}\right)=\left\{e_{i}=v_{i} v_{i+1}, e_{j}^{\prime}=u_{j} v_{j}: 1 \leq i \leq n-1,1 \leq j \leq n\right\}$. Define $f: V\left(P_{n} \odot K_{1}\right) \rightarrow\{1,2,3, . ., p q\}$ is as follows:

$f\left(v_{1}\right)=1, f\left(v_{i}\right)=f\left(v_{i-1}\right)+2(-1)^{i} T_{2(n-i+1)}$ for $2 \leq i \leq n, f\left(u_{1}\right)=2 T_{2 n-1}$, $f\left(u_{i}\right)=f\left(v_{i}\right)+2(-1)^{i+1} T_{2(n-i)+1}$ for $2 \leq i \leq n$.

For the vertex label $f$, the induced edge label $f^{*}$ is as follows:

$f^{*}\left(e_{i}\right)=T_{2(n-i)}$ for $1 \leq i \leq n-1, f^{*}\left(e_{j}^{\prime}\right)=T_{2(n-j)+1}$ for $1 \leq j \leq n$.

We can see that the edge labels are the triangular numbers: $T_{1}, T_{2}, \ldots, T_{2 n-1}$. Hence $P_{n} \odot K_{1}$ is a restricted triangular difference mean graph. The restricted triangular difference mean labeling of $P_{5} \odot K_{1}$ is shown in Figure 3 . 


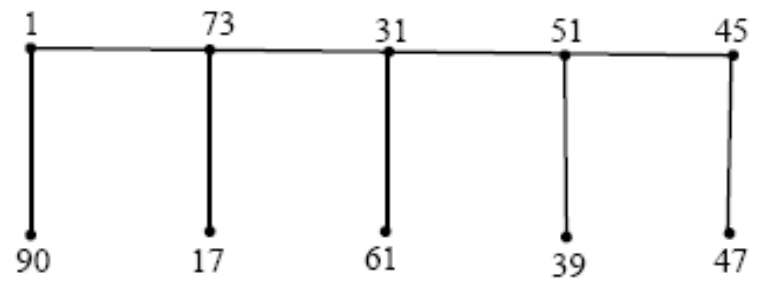

Figure 3

Theorem 2.4. A necessary condition for a graph to be a restricted triangular difference mean graph is that $p>q$.

Proof. Suppose that the graph $G=(V, E)$ with $p$ vertices and $q$ edges is a restricted triangular difference mean graph.

Suppose $p \leq q$.

Let $f: V(G) \rightarrow\{1,2,3, \ldots, p q\}$ be a restricted triangular difference mean labeling of $G$. Then the induced edge labels are $\left\{T_{1}, T_{2}, \ldots, T_{q}\right\}$.

Let $u$ and $v$ be any two adjacent vertices of $\mathrm{G}$ such that $f^{*}(u v)=T_{q}$.

Without loss of generality, we assume $f(u)>f(v)$.

Then $1 \leq f(u), f(v) \leq p q$.

Case(i). Suppose $|f(u)-f(v)|$ is odd.

$$
\begin{gathered}
\frac{|f(u)-f(v)|+1}{2}=T_{q}=\frac{q(q+1)}{2} \\
f(u)-f(v)=q^{2}+q-1 \\
f(u)=f(v)+q^{2}+q-1 .
\end{gathered}
$$

Now, $p \leq q$

$$
\begin{gathered}
p q \leq q^{2} \\
p q \leq q^{2}<q^{2}+q \\
f(u) \leq p q<q^{2}+q \\
f(v)+q^{2}+q-1<q^{2}+q \\
f(v)-1<0
\end{gathered}
$$


$f(v)<1$, which is a contradiction.

Case(ii). Suppose $|f(u)-f(v)|$ is even.

$$
\begin{gathered}
\frac{|f(u)-f(v)|}{2}=T_{q}=\frac{q(q+1)}{2} \\
f(u)-f(v)=q^{2}+q \\
f(v)+q^{2}+q<q^{2}+q .
\end{gathered}
$$

$f(v)<0$, which is a contradiction.

Theorem 2.5. The complete graph $K_{n}(n \geq 1)$ is a restricted triangular difference mean graph if and only if or $n<3$.

Proof. Let $V\left(K_{n}\right)=\left\{v_{i}: 1 \leq i \leq n\right\}$ and $E\left(K_{n}\right)=\left\{v_{i} v_{i+1}: 1 \leq i \leq\right.$ $n-1\} \cup\left\{v_{2} v_{i+2}: 1 \leq i \leq n-2\right\} \cup\left\{v_{3} v_{i+3}: 1 \leq i \leq n-3\right\} \cup \ldots \cup\left\{v_{n-1} v_{n}\right\}$.

The graph $K_{n}$ has $n$ vertices and $\left(\begin{array}{c}n \\ 2\end{array}\right)$ edges.

When $n<3$, the result is obvious.

Conversely, suppose $K_{n}$ is a restricted triangular difference mean graph.

Suppose $n \geq 3$.

Let $f: V\left(K_{n}\right) \rightarrow\left\{1,2,3, \ldots, p q=n\left(\begin{array}{c}n \\ 2\end{array}\right)\right\}$ be defined as follows:

Let $u$ and $v$ be any two adjacent vertices of $\mathrm{G}$ such that $f^{*}(u v)=T_{q}$.

Let $e=u v$ be labeled with $\left(\begin{array}{c}n \\ 2\end{array}\right)$.

Now $1 \leq f(u), f(v) \leq n\left(\begin{array}{c}n \\ 2\end{array}\right)$.

Then either $\frac{|f(u)-f(v)|}{2}=T\left(\begin{array}{c}n \\ 2\end{array}\right)$ or $\frac{|f(u)-f(v)|+1}{2}=T\left(\begin{array}{c}n \\ 2\end{array}\right)$

Without loss of generality we assume $f(u)>f(v)$. 


$$
\begin{aligned}
f(u) & =\left(\begin{array}{l}
n \\
2
\end{array}\right)\left[\left(\begin{array}{l}
n \\
2
\end{array}\right)+1\right]+f(v) \\
\geq & \left(\begin{array}{l}
n \\
2
\end{array}\right)\left[\left(\begin{array}{l}
n \\
2
\end{array}\right)+1\right]+1 \\
& >\left(\begin{array}{l}
n \\
2
\end{array}\right)\left[\left(\begin{array}{l}
n \\
2
\end{array}\right)+1\right]
\end{aligned}
$$

Thus, we have

$$
\begin{gathered}
\left(\begin{array}{c}
n \\
2
\end{array}\right)\left[\left(\begin{array}{l}
n \\
2
\end{array}\right)+1\right]<f(u) \leq n\left(\begin{array}{l}
n \\
2
\end{array}\right) \\
{\left[\left(\begin{array}{l}
n \\
2
\end{array}\right)+1\right]<n,}
\end{gathered}
$$

This is a contradiction.

or

$$
\begin{gathered}
f(u)=\left(\begin{array}{l}
n \\
2
\end{array}\right) \text { or }\left[\left(\begin{array}{l}
n \\
2
\end{array}\right)+1\right]+f(v)-1 \\
\geq\left(\begin{array}{l}
n \\
2
\end{array}\right)\left[\left(\begin{array}{l}
n \\
2
\end{array}\right)+1\right] .
\end{gathered}
$$

Then we have $\left(\begin{array}{c}n \\ 2\end{array}\right)\left[\left(\begin{array}{c}n \\ 2\end{array}\right)+1\right] \leq f(u) \leq n\left(\begin{array}{c}n \\ 2\end{array}\right)$

$$
\left[\left(\begin{array}{c}
n \\
2
\end{array}\right)+1\right] \leq n, \quad \text { which implies } n \leq 2 \text {. }
$$

This is also a contradiction.

Theorem 2.6. The bistar $B_{m, n}(m \geq 1, n \geq 1)$ is a restricted triangular difference mean graph.

Proof. Let $V\left(B_{m, n}\right)=\left\{u, v, u_{i}, v_{j}: 1 \leq i \leq m, 1 \leq j \leq n\right\}$ and $E\left(B_{m, n}\right)=\left\{u v, u u_{i}, v v_{j}: 1 \leq i \leq m, 1 \leq j \leq n\right\}$.

Define $f: V\left(B_{m, n}\right) \rightarrow\{1,2,3, \ldots, p q\}$ is as follows:

$f(u)=1, f(v)=3, f\left(u_{1}\right)=2 T_{m+n+1}$,

$f\left(u_{i}\right)=2 T_{m+n-i+2}+1$ for $2 \leq i \leq m, f\left(v_{j}\right)=2 T_{j+1}+3$ for $1 \leq j \leq n$.

For the vertex label $f$, the induced edge label $f^{*}$ is as follows: 
$f^{*}(u v)=T_{1}, f^{*}\left(u u_{i}\right)=T_{n+m-i+2}$ for $1 \leq i \leq m$ and $f^{*}\left(v v_{j}\right)=T_{j+1}$ for $1 \leq j \leq n$.

Hence, the induced edge labels are the first $m+n+1$ triangular numbers. Therefore, $B_{m, n}$ is a restricted triangular difference mean graph.

The restricted triangular difference mean labeling of $B_{4,5}$ is shown in Figure 4 .

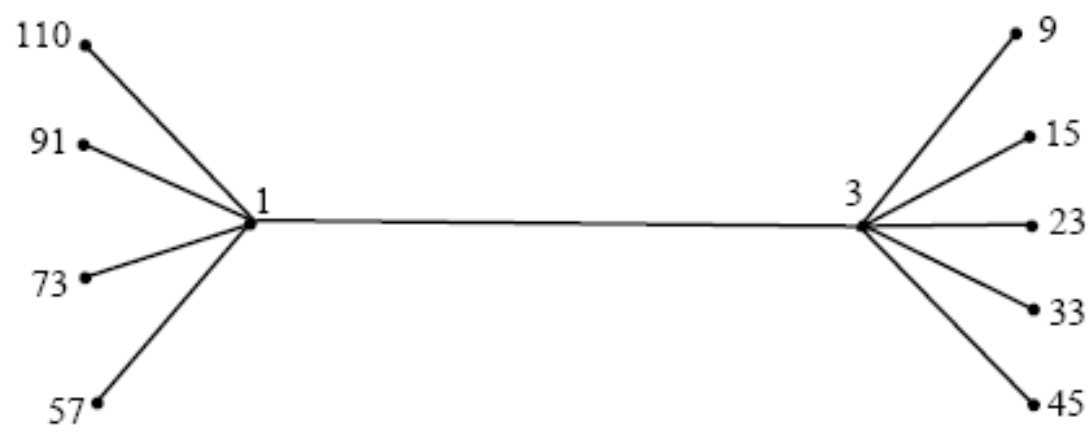

Figure 4

Theorem 2.7. A graph obtained by joining the roots of different stars to a new vertex, is a restricted triangular difference mean graph.

Proof. Let $K_{1, n_{1}}, K_{1, n_{2}}, \ldots, K_{1, n_{k}}$ be a $k$ stars. Let $G$ be a graph obtained by joining the central vertices of the stars to the new vertex $u$.

Let $n=n_{1}+n_{2}+\ldots+n_{k}$.

Assign 1 to $u ; 2 T_{n+k}, 2 T_{n+k-1}, \ldots, 2 T_{n+1}$ to the central vertices of the stars. $2 T_{n+k}-2 T_{1}, 2 T_{n+k}-2 T_{2}, \ldots,+1,2 T_{n+k}-2 T_{n_{1}}$ to the pendant vertices of the first star; $+1,2 T_{n+k-1}-2 T_{n_{1}+1},+1,2 T_{n+k-1}-2 T_{n_{1}+2}, \ldots, 2 T_{n+k-1}-2 T_{n_{1}+n_{2}}$ to the pendant vertices of the second star; and so on finally assign the numbers $+1,2 T_{n+1}-2 T_{n_{1}+n_{2}+\ldots+n_{k-1}+1},+1,2 T_{n+1}-2 T_{n_{1}+n_{2}+\ldots+n_{k-1}+2}, \ldots$, $+1,2 T_{n+1}-2 T_{n_{1}+n_{2}+\ldots+n_{k-1}+n_{k}}$ to the pendant vertices of the last star. We observe that the edge labels are the triangular numbers $T_{1}, T_{2}, \ldots$, $T_{k+n_{1}+n_{2}+\ldots+n_{k-1}+n_{k}}$, also the vertex labels are all different. The restricted triangular difference mean labeling of the tree given in Theorem 2.7 with $k=3, n_{1}=4, n_{2}=5$ and $n_{3}=4$ is shown in Figure 5 . 


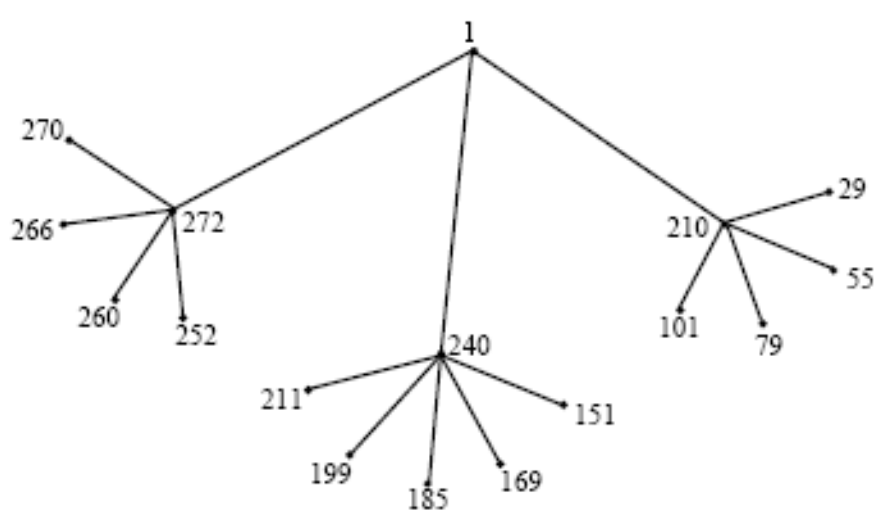

Figure 5

Theorem 2.8. The caterpillar $S\left(n_{1}, n_{2}, \ldots, n_{m}\right)$ is a restricted triangular difference mean graph.

Proof. Let $v_{1}, v_{2}, \ldots, v_{m}$ be the vertices of the path $P_{m}$ and $v_{i}^{j}(1 \leq i \leq$ $\left.n_{j}, 1 \leq j \leq m\right)$ be the pendant vertices incident with $v_{j}(1 \leq j \leq m)$.

Let $n=n_{1}+n_{2}+\ldots+n_{m}$.

Then $V\left(S\left(n_{1}, n_{2}, \ldots, n_{m}\right)\right)=\left\{v_{j}, v_{i}^{j}: 1 \leq i \leq n_{j}, 1 \leq j \leq m\right\}$ and

$E\left(S\left(n_{1}, n_{2}, \ldots, n_{m}\right)\right)=\left\{v_{t} v_{t+1}, v_{j} v_{i}^{j}: 1 \leq t \leq m-1,1 \leq i \leq n_{j}, 1 \leq j \leq\right.$ $m\}$.

Define $f: V\left(S\left(n_{1}, n_{2}, \ldots, n_{m}\right)\right) \rightarrow\{1,2,3, \ldots, p q\}$ is as follows:

$f\left(v_{1}\right)=1, f\left(v_{j}\right)=2\left(T_{1}+T_{2}+\ldots+T_{j-1}\right)+1$ for $2 \leq j \leq m$, $f\left(v_{i}^{1}\right)=2 T_{n+m-i}$ for $1 \leq i \leq n_{1}$,

$f\left(v_{i}^{j}\right)=2\left(T_{1}+T_{2}+\ldots+T_{j-1}\right)+2 T_{m+n-n_{1}-n_{2}-\ldots-n_{j-1}-i}+1$ for $2 \leq j \leq m$ and $1 \leq i \leq n_{j}$.

For each vertex label $f$, the induced edge label $f^{*}$ is as follows:

$f^{*}\left(v_{j} v_{j+1}\right)=T_{j}$ for $1 \leq j \leq m-1$,

$f^{*}\left(v_{1} v_{i}^{1}\right)=T_{m+n-i}$,

$f^{*}\left(v_{j} v_{i}^{j}\right)=T_{m+n-n_{1}-n_{2}-\ldots-n_{j-1}-i}$ for $2 \leq j \leq m$ and $1 \leq i \leq n_{j}$.

Then the edge labels are the triangular numbers $T_{1}, T_{2}, \ldots, T_{m-1}$,

$T_{m}, \ldots, T_{m-1+n_{1}+\ldots+n_{m}}$ and also the vertex labels are different.

Hence $S\left(n_{1}, n_{2}, \ldots, n_{m}\right)$ is a restricted triangular difference mean graph.

The restricted triangular difference mean labeling of $S(3,5,4,6)$ is shown in Figure 6. 


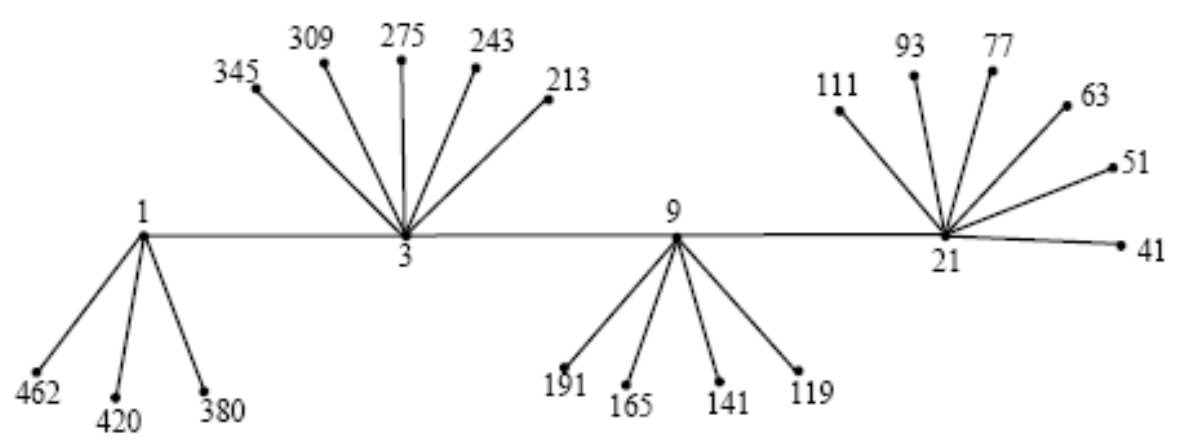

Figure 6

Theorem 2.9. The complete bipartite graph $K_{m, n}$ is a restricted triangular difference mean graph if and only if $n<2$ or $m<2$.

Proof. If $m=n=1$, then the graph $K_{1,1}=K_{2}$ which is a restricted triangular difference mean graph.

If $m=1$ or $r=1, K_{m, n}$ is a star graph and hence it is a restricted triangular difference mean graph.

Conversely suppose that $m, n \geq 2$.

Let $G$ be a restricted triangular difference mean graph $K_{m, n}$.

Let $V(G)=\left\{u_{i}, v_{j}: 1 \leq i \leq m, 1 \leq j \leq n\right\}$ and $E(G)=\left\{u_{i} v_{j}: 1 \leq i \leq\right.$ $m, 1 \leq j \leq n\}$. Then $G$ has $m+n$ vertices and $m n$ edges.

Let $f: V(G) \rightarrow\{1,2,3, \ldots, p q=m n(m+n)\}$ be defined as follows:

Now $1 \leq f\left(u_{i}\right), f\left(v_{j}\right) \leq m n(m+n)$ for all $u_{i}$ and $v_{j}$.

Let $e=u_{i} v_{j}$ be labeled with $T_{m n}$.

Without loss of generality, let $f\left(u_{i}\right)>f\left(v_{j}\right)$.

Case(i).

Suppose $\frac{\left|f\left(u_{i}\right)-f\left(v_{j}\right)\right|}{2}=T_{m n}=\frac{m n(m n+1)}{2}$

Then 


$$
\begin{gathered}
f\left(u_{i}\right)=f\left(v_{j}\right)+m n(m n+1) \\
\geq 1+m n(m n+1) \\
>m n(m n+1) .
\end{gathered}
$$

That is, we have

$$
\begin{gathered}
m n(m n+1)<f\left(u_{i}\right) \leq m n(m+n) \\
m n+1<m+n \text { for all } m, n \geq 2,
\end{gathered}
$$

which is a contradiction.

\section{Case(ii).}

Suppose $\frac{\left|f\left(u_{i}\right)-f\left(v_{j}\right)\right|+1}{2}=T_{m n}=\frac{m n(m n+1)}{2}$

Then

$$
\begin{aligned}
f\left(u_{i}\right)= & f\left(v_{j}\right)+m n(m n+1)-1 \\
& \geq m n(m n+1) .
\end{aligned}
$$

If $m n(m n+1) \leq f\left(u_{i}\right) \leq m n(m+n)$, then $m n+1 \leq m+n$ for all $m, n \geq 2$, which is a contradiction.

Theorem 2.10. The wheel graph $W_{n}, n>2$ is not a restricted triangular difference mean graph.

Proof. Let $G$ be a wheel graph $W_{n}, n>2$.

Let $V(G)=\left\{v, v_{i}: 1 \leq i \leq n\right\}$ and $E(G)=\left\{v v_{i}, v_{j} v_{j+1}: 1 \leq i \leq n, 1 \leq\right.$ $j \leq n-1\} \cup\left\{v_{n} v_{1}\right\}$.

Then the graph $G$ has $n+1$ vertices and $2 n$ edges.

Let $f: V(G) \rightarrow\{1,2,3, \ldots, p q=2 n(n+1)\}$ be defined as follows:

Suppose $G$ is a restricted triangular difference mean graph.

Let $f(v)=a$ and $f\left(v_{i}\right)=b$ for some $i$.

Suppose $a>b$. Then $1 \leq b<a \leq 2 n(n+1)$.

Now we consider two cases.

Case(i).

Suppose $\frac{\left|f(v)-f\left(v_{i}\right)\right|}{2}=T_{2 n}=\frac{2 n(2 n+1)}{2}$ 


$$
\begin{gathered}
\frac{a-b}{2}=\frac{2 n(2 n+1)}{2} \\
a=2 n(2 n+1)+b \\
\geq 2 n(2 n+1)+1 \\
>2 n(2 n+1) \\
>2 n(n+1),
\end{gathered}
$$

which is a contradiction.

\section{Case(ii).}

Suppose $\frac{\left|f(v)-f\left(v_{i}\right)\right|+1}{2}=T_{2 n}=\frac{2 n(2 n+1)}{2}$

$$
\begin{aligned}
& a-b+1=2 n(2 n+1) \\
& a=2 n(2 n+1)+b-1 \\
& \geq 2 n(2 n+1) \\
& >2 n(n+1),
\end{aligned}
$$

which is also a contradiction.

Hence the wheel graph $W_{n}, n>2$ is not a restricted triangular difference mean graph.

\section{References}

[1] F. Harary, Graph theory. Reading, MA: Addison-Wesley, 1972.

[2] J. Gallian, "A dynamic survey of graph labeling", 22th ed. The electronics journal of combinatorics, vol. \# DS6, p. 535, 2019, doi: 10.37236/27

[3] P. Jeyanthi, M. Selvi, and D. Ramya, "Triangular difference mean graphs", International journal of mathematical combinatorics, to appear. 